\title{
Electronic transport in a one-dimensional random array of scatterers
}

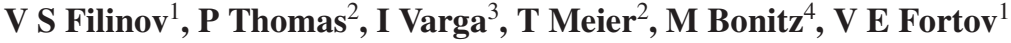 \\ and $\mathrm{S} \mathbf{W} \mathrm{Koch}^{2}$ \\ ${ }^{1}$ Institute for High Energy Density, Russian Academy of Sciences, Izhorskay 13/19, \\ Moscow 127412, Russia \\ ${ }^{2}$ Fachbereich Physik und Wiss. Zentrum für Materialwissenschaften, \\ Philipps-Universität Marburg, D-35032 Marburg, Germany \\ ${ }^{3}$ Elméleti Fizika Tanszék, Budapesti Múszaki és Gazdaságtudományi Egyetem, \\ H-1521 Budapest, Hungary \\ ${ }^{4}$ Fachbereich Physik, Universität Rostock, Universitätsplatz 3, D-18051 Rostock, Germany \\ E-mail: filinov@ok.ru
}

Received 16 December 2002, in final form 13 February 2003

Published 22 May 2003

Online at stacks.iop.org/JPhysA/36/5905

\begin{abstract}
The quantum dynamics of an ensemble of interacting electrons in an array of random scatterers is treated using a new numerical approach for the calculation of average values of quantum operators and time correlation functions in the Wigner representation. This approach combines both molecular dynamics and Monte Carlo methods and computes numerical traces and spectra of the relevant dynamical quantities such as momentum-momentum correlation functions and spatial dispersions. Considering, as an application, a system with fixed scatterers, the results clearly demonstrate that the many-particle interaction between the electrons can lead to an enhancement of the conductivity at intermediate densities.
\end{abstract}

PACS numbers: 72.15.Rn, 61.43.-j, 05.30.-d

Non-interacting electrons in an array of fixed random scatterers are known to experience Anderson localization at temperature $T=0$ in one-dimensional systems [1-3]. However, it is expected that the many-particle interaction leads to delocalization tendencies which have been confirmed for simple models $[4,5]$. The purpose of this paper is twofold. (i) We present a novel approach which does not rely on model interactions and small system sizes. It can be applied to a wide variety of physical systems, such as plasmas, liquids and solids. (ii) As an illustration we investigate the influence of the many-particle interaction on electronic transport in a one-dimensional disordered array of scatterers interacting repulsively with the electron system. With electron-electron interaction neglected, such a system shows Anderson localization. It is the purpose of this application to study the effect of the true long-range electron-electron Coulomb interaction on the mobility of the electrons. 
To study the influence of these effects on kinetic electron properties in a random environment we have simulated the quantum dynamics in a one-dimensional canonical ensemble at finite temperature for both interacting and non-interacting electrons using a quantum-dynamics-Monte Carlo scheme. The main quantities calculated in this paper are the temporal momentum-momentum correlation functions and their frequency-domain Fourier transforms. We discovered that the results strongly depend on the electron-electron interaction, clearly demonstrating the delocalizing influence of the many-particle interaction at densities around $R_{s}=\bar{r} / a_{0}=5\left(\bar{r}\right.$ is the mean interparticle distance and $a_{0}$ is the effective Bohr radius) even at finite temperatures. Our approach also treats the positions of the scattering centres as dynamical variables. We are, therefore, able to generate various initial conditions.

\section{Wigner representation of time correlation functions}

According to the Kubo formula the conductivity is the Fourier transform of the current-current correlation function. Our starting point is the general operator expression for the canonical ensemble-averaged time correlation function [6]:

$$
C_{F A}(t)=Z^{-1} \operatorname{Tr}\left\{\hat{F} \mathrm{e}^{\mathrm{i} \hat{H} t_{c}^{*} / \hbar} \hat{A} \mathrm{e}^{-\mathrm{i} \hat{H} t_{c} / \hbar}\right\}
$$

where $\hat{H}$ is the Hamiltonian of the system expressed as a sum of the kinetic energy operator, $\hat{K}$, and the potential energy operator, $\hat{U}$. Time is taken to be a complex quantity, $t_{c}=t-\mathrm{i} \hbar \beta / 2$, where $\beta=1 / k_{B} T$ is the inverse temperature with $k_{B}$ denoting the Boltzmann constant. The operators $\hat{F}$ and $\hat{A}$ are quantum operators of the dynamic quantities under consideration and $Z=\operatorname{Tr}\left\{\mathrm{e}^{-\beta \hat{H}}\right\}$ is the partition function. The Wigner representation of the time correlation function in a $v$-dimensional space can be written as

$$
C_{F A}(t)=(2 \pi \hbar)^{-2 v} \iint \mathrm{d} \mu_{1} \mathrm{~d} \mu_{2} F\left(\mu_{1}\right) A\left(\mu_{2}\right) W\left(\mu_{1} ; \mu_{2} ; t ; \mathrm{i} \hbar \beta\right)
$$

where we introduce the short-hand notation for the phase space point, $\mu_{i}=\left(p_{i}, q_{i}\right),(i=1,2)$, and $p$ and $q$ comprise the momenta and coordinates, respectively, of all particles in the system. $W\left(\mu_{1} ; \mu_{2} ; t ; i \hbar \beta\right)$ is the spectral density expressed as

$$
\begin{aligned}
& W\left(\mu_{1} ; \mu_{2} ; t ; \mathrm{i} \hbar \beta\right)=Z^{-1} \iint \mathrm{d} \xi_{1} \mathrm{~d} \xi_{2} \mathrm{e}^{\mathrm{i} \frac{p_{1} \xi_{1}}{\hbar}} \mathrm{e}^{\mathrm{i} \frac{p_{2} \xi_{2}}{\hbar}} \\
& \times\left\langle q_{1}+\frac{\xi_{1}}{2}\left|\mathrm{e}^{\mathrm{i} \hat{H} t_{c}^{*} / \hbar}\right| q_{2}-\frac{\xi_{2}}{2}\right\rangle\left\langle q_{2}+\frac{\xi_{2}}{2}\left|\mathrm{e}^{-\mathrm{i} \hat{H} t_{c} / \hbar}\right| q_{1}-\frac{\xi_{1}}{2}\right\rangle
\end{aligned}
$$

and $A(\mu)$ denotes Weyl's symbol [7] of operator $\hat{A}: A(\mu)=\int \mathrm{d} \xi \mathrm{e}^{-\mathrm{i} \frac{p \xi}{h}}\left\langle q-\frac{\xi}{2}|\hat{A}| q+\frac{\xi}{2}\right\rangle$, and similarly for the operator $\hat{F}$. Hence, the problem of the numerical calculation of the canonically averaged time correlation function is reduced to the computation of the spectral density.

To obtain the integral equation for $W$ let us introduce a pair of dynamic $p, q$-trajectories $\left\{\bar{q}_{\tau}\left(\tau ; p_{1}, q_{1}, t\right), \bar{p}_{\tau}\left(\tau ; p_{1}, q_{1}, t\right)\right\}$ and $\left\{\tilde{q}_{\tau}\left(\tau ; p_{2}, q_{2}, t\right), \tilde{p}_{\tau}\left(\tau ; p_{2}, q_{2}, t\right)\right\}$ starting at $\tau=t$ from the initial condition $\left\{q_{1}, p_{1}\right\}$ and $\left\{q_{2}, p_{2}\right\}$ propagating in 'negative' and 'positive' time directions, respectively:

$$
\begin{aligned}
& \frac{\mathrm{d} \bar{p}_{\tau}}{\mathrm{d} \tau}=\frac{1}{2} F\left[\bar{q}_{\tau}(\tau)\right], \frac{\mathrm{d} \bar{q}_{\tau}}{\mathrm{d} \tau}=\frac{\bar{p}_{\tau}(\tau)}{2 m} \\
& \text { with } \quad \bar{p}_{t}\left(\tau=t ; p_{1}, q_{1}, t\right)=p_{1}, \quad \bar{q}_{t}\left(\tau=t ; p_{1}, q_{1}, t\right)=q_{1}
\end{aligned}
$$


and

$$
\begin{aligned}
& \frac{\mathrm{d} \tilde{p}_{\tau}}{\mathrm{d} \tau}=-\frac{1}{2} F\left[\tilde{q}_{\tau}(\tau)\right], \quad \frac{\mathrm{d} \tilde{q}_{\tau}}{\mathrm{d} \tau}=-\frac{\tilde{p}_{\tau}(\tau)}{2 m} \\
& \text { with } \quad \bar{p}_{t}\left(\tau=t ; p_{1}, q_{1}, t\right)=p_{1}, \quad \tilde{q}_{t}\left(\tau=t ; p_{1}, q_{1}, t\right)=q_{1},
\end{aligned}
$$

where $F(q) \equiv-\nabla \tilde{U}$ with $\tilde{U}$ being the total potential, i.e. the sum of all pair interactions $U_{a b}$. Then, as has been proved in [8], $W$ obeys the following integral equation:

$W\left(\mu_{1} ; \mu_{2} ; t ; \mathrm{i} \hbar \beta\right)=\bar{W}\left(\bar{p}_{0}, \bar{q}_{0} ; \tilde{p}_{0}, \tilde{q}_{0} ; \mathrm{i} \hbar \beta\right)$

$$
\begin{aligned}
& +\frac{1}{2} \int_{0}^{t} \mathrm{~d} \tau \int \mathrm{d} s W\left(\bar{p}_{\tau}-s, \bar{q}_{\tau} ; \tilde{p}_{\tau}, \tilde{q}_{\tau} ; \tau ; \mathrm{i} \hbar \beta\right) \varpi\left(s, \bar{q}_{\tau}\right) \\
& -\frac{1}{2} \int_{0}^{t} \mathrm{~d} \tau \int \mathrm{d} s W\left(\bar{p}_{\tau}, \bar{q}_{\tau} ; \tilde{p}_{\tau}-s, \tilde{q}_{\tau} ; \tau ; \mathrm{i} \hbar \beta\right) \varpi\left(s, \tilde{q}_{\tau}\right)
\end{aligned}
$$

where $\varpi(s, q)=\frac{4}{(2 \pi \hbar)^{2} \hbar} \int \mathrm{d} q^{\prime} \tilde{U}\left(q-q^{\prime}\right) \sin \left(\frac{2 s q^{\prime}}{\hbar}\right)+F(q) \nabla \delta(s)$, and $\delta(s)$ is the Dirac delta function. Equation (4) has to be supplemented by an initial condition for the spectral density at $t=0: W\left(\mu_{1} ; \mu_{2} ; 0 ; \mathrm{i} \hbar \beta\right)=\left.\bar{W}\left(\mu_{1} ; \mu_{2} ; \mathrm{i} \hbar \beta\right)\right|_{t=0} \equiv \bar{W}$. The $\tau$-integrals connect the points $\bar{p}_{\tau}, \bar{q}_{\tau} ; \tilde{p}_{\tau}, \tilde{q}_{\tau}$ at time $\tau$ of the above mentioned dynamic $p, q$-trajectories with the points $p_{1}, q_{1} ; p_{2}, q_{2}$ at time $t$ whereas in $\bar{W}$ the trajectories are to be taken at $\tau=0$ (denoted by the subscript ' 0 '). The function $\bar{W}$ can be expressed in the form of a finite difference approximation of the path integral [8-10]: $\bar{W}\left(\mu_{1} ; \mu_{2} ; \mathrm{i} \hbar \beta\right) \approx$ $\iint \mathrm{d} \tilde{q}_{1} \cdots \mathrm{d} \tilde{q}_{n} \iint \mathrm{d} q_{1}^{\prime} \cdots \mathrm{d} q_{n}^{\prime} \Psi\left(\mu_{1} ; \mu_{2} ; \tilde{q}_{1}, \ldots, \tilde{q}_{n} ; q_{1}^{\prime}, \ldots, q_{n}^{\prime} ; \mathrm{i} \hbar \beta\right)$, with

$$
\begin{aligned}
\Psi\left(\mu_{1} ; \mu_{2} ; \tilde{q}_{1},\right. & \left.\ldots, \tilde{q}_{n} ; q_{1}^{\prime}, \ldots, q_{n}^{\prime} \mathrm{i} \hbar \beta\right) \\
\equiv & \frac{1}{Z}\left\langle q_{1}\left|\mathrm{e}^{-\epsilon \hat{K}}\right| \tilde{q}_{1}\right\rangle \mathrm{e}^{-\epsilon U\left(\tilde{q}_{1}\right)}\left\langle\tilde{q}_{1}\left|\mathrm{e}^{-\epsilon \hat{K}}\right| \tilde{q}_{2}\right\rangle \mathrm{e}^{-\epsilon U\left(\tilde{q}_{2}\right)} \\
& \cdots \mathrm{e}^{-\epsilon U\left(\tilde{q}_{n}\right)}\left\langle\tilde{q}_{n}\left|\mathrm{e}^{-\epsilon \hat{K}}\right| q_{2}\right\rangle \varphi\left(p_{2} ; \tilde{q}_{n}, q_{1}^{\prime}\right) \\
& \times\left\langle q_{2}\left|\mathrm{e}^{-\epsilon \hat{K}}\right| q_{1}^{\prime}\right\rangle \mathrm{e}^{-\epsilon U\left(q_{1}^{\prime}\right)}\left\langle q_{1}^{\prime}\left|\mathrm{e}^{-\epsilon \hat{K}}\right| q_{2}^{\prime}\right\rangle \mathrm{e}^{-\epsilon U\left(q_{2}^{\prime}\right)} \\
& \cdots \mathrm{e}^{-\epsilon U\left(q_{n}^{\prime}\right)}\left\langle q_{n}^{\prime}\left|\mathrm{e}^{-\epsilon \hat{K}}\right| q_{1}\right\rangle \varphi\left(p_{1} ; q_{n}^{\prime}, \tilde{q}_{1}\right)
\end{aligned}
$$

where $\varphi\left(p ; q^{\prime}, q^{\prime \prime}\right) \equiv\left(2 \lambda^{2}\right)^{v / 2} \exp \left[-\frac{1}{2 \pi}\left\langle\frac{p \lambda}{\hbar}+\mathrm{i} \pi \frac{q^{\prime}-q^{\prime \prime}}{\lambda} \mid \frac{p \lambda}{\hbar}+\mathrm{i} \pi \frac{q^{\prime}-q^{\prime \prime}}{\lambda}\right\rangle\right]$, and $\langle x \mid y\rangle$ denotes the scalar product of two vectors $\mathbf{x}, \mathbf{y}$. In this expression the original (unknown) density matrix of the correlated system $\mathrm{e}^{-\beta(\hat{K}+\hat{U})}$ has been decomposed into $2 n$ factors, each at a $2 n$ times higher temperature, with the inverse $\epsilon=\beta / 2 n$ and the corresponding high temperature de Broglie wavelength squared $\lambda^{2} \equiv 2 \pi \hbar^{2} \epsilon / m$. This leads to a product of known high-temperature (weakly correlated) density matrices, however, at the price of $2 n$ additional integrations over the intermediate coordinate vectors (over the 'path'). This representation is exact in the limit $n \rightarrow \infty$, and, for finite $n$, an error of order $1 / n$ occurs. The function $\Psi$ has to be generalized to properly account for spin-statics effects. This gives rise to an additional spin part of the density matrix and antisymmetrization of one off-diagonal matrix element. To improve the accuracy of the obtained expression, we will replace $U_{a b} \rightarrow U_{a b}^{\text {eff }}$ where $U_{a b}^{\text {eff }}$ is the proper effective quantum pair potential, see below. For more details on the path integral concept, we refer to $[11,12]^{5}$.

\section{Quantum dynamics}

Let us now come back to the integral equation (4). For the discussion we note that the integral equation (4) can be exactly converted into an iteration series (which is obtained by successively

\footnotetext{
5 Details on our direct fermionic path integral Monte Carlo simulations are given in [13].
} 
replacing $W \rightarrow \bar{W}$ under the integrals). This series is, however, not a perturbative expansion in the interaction, neither in the electron-scatterer nor in the electron-electron interaction. It is rather an expansion in terms of corrections to classical trajectories of fully interacting electrons and electrons with scatterers. So multiple scattering effects are fully included. Physically the second order and other terms of the iteration series include corrections to the classical electron trajectories (momentum jumps related to the uncertainty principle between momentum-coordinate and energy-time) and corrections to the quantum Wigner-Liouville function. A detailed investigation of the conditions for which the contribution of the next terms of the iteration series should be taken into account is presented in [8, 14-16].

As mentioned above, the first term $\bar{W}$ describes propagation of a correlated quantum initial state along the characteristics of the classical Wigner-Liouville equation. This term, containing all powers of Planck's constant, is the coherent sum of complex-valued contributions of a trajectory ensemble related to $\bar{W}$. This term allows us to describe quantum coherent effects such as Anderson localization, while the other terms of the iteration series describe deviations from the classical trajectories: the trajectories are perturbed by finite momentum jumps $s$ occurring at arbitrary times $\tau, 0 \leqslant \tau \leqslant t$ [9]. These terms are essential for the recovery of tunnelling effects, we expect that they do not give dominant contributions to coherence and localization phenomena. With increasing quantum degeneracy (i.e. decreasing temperature or/and increasing density) the magnitude of these terms will grow. However, our numerical results below refer to finite temperature and moderate degeneracy $n_{1 \mathrm{D}} \lambda_{e e}=0.2 \ldots 7$. We, therefore, will include in the following numerical analysis only the first term of equation (4).

As an application, in this work we will consider a system composed of heavy particles (called scatterers) with mass $m_{s}$ and negatively charged electrons with mass $m_{e}$. To avoid bound state effects due to attraction we consider in this case study only negatively charged scatterers, assuming a positive background for charge neutrality. The influence of electronscatterer attraction will be studied in a further publication.

The developed Monte Carlo approach, based on ideas [8, 14, 15, 17], allows us to generate, in a controlled way, various kinds of quantum dynamics and initial conditions of the manybody system, in particular, (i) those which are characteristic of the fully interacting system (i.e. including scatterer-scatterer ( $\mathrm{s}-\mathrm{s})$, electron-scatterer (e-s) and electron-electron (e-e)) and (ii) those which result if some aspects of these interactions are ignored.

\section{Numerical results}

We now apply the numerical approach, explained above, to the problem of an interacting ensemble of electrons and disordered scatterers in one dimension. In all calculations, times, frequencies and distances are measured in atomic units. The average distance between electrons, $R_{s}=1 /\left(n_{e} a_{0}\right)$, was varied between 12.0 and 0.55 , with the densities of electrons and heavy scatterers taken to be equal. The results obtained were practically insensitive to the variation of the whole number of the particles in MC cell from 30 up to 50 and also of the number of high temperature density matrices (determined by the number of factors $n$ ), ranging from 10 to 20 . Estimates of the average statistical error gave the value of the order 5-7\%. We studied two different temperatures: $k_{B} T /\left|V_{0}^{e s}\right|=0.45$ and 0.28 , corresponding to $\lambda_{e e} / a_{0} \sim 2.2$ and $\lambda_{e e} / a_{0} \sim 3.5$, respectively. The strengths of the three interactions in the system are fixed arbitrarily at the ratio $V_{0}^{e e}: V_{0}^{e s}: V_{0}^{s s} \sim 0.7: 1: 32$.

According to the Kubo formula [6] our calculations include two different stages: (i) generation of the initial conditions (configuration of scatterers and electrons) in the canonical ensemble with probability proportional to the quantum density matrix and (ii) generation of the dynamic trajectories on the time scale $t^{\prime}$ in phase space, starting from these initial 

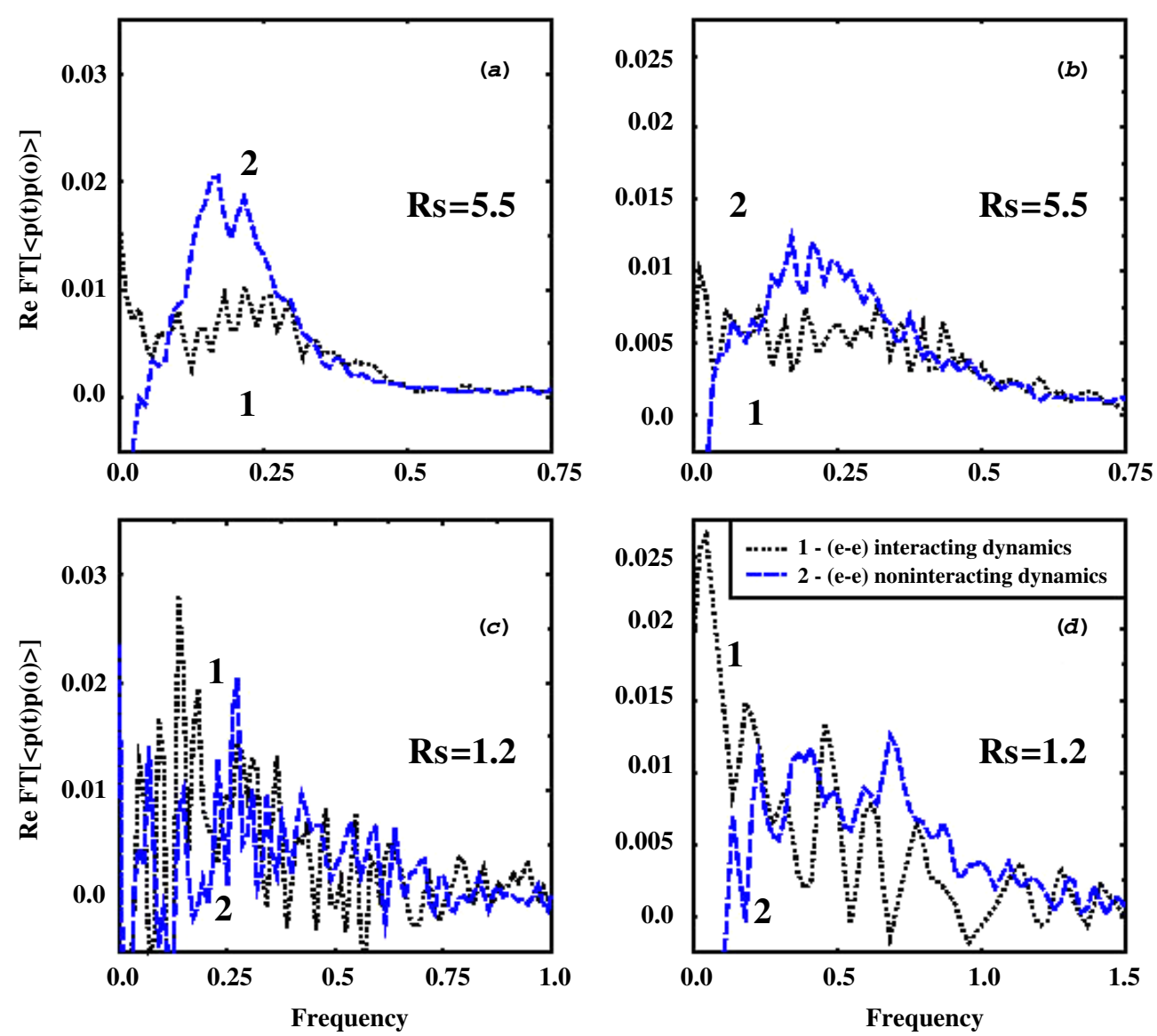

Figure 1. Real part of the Fourier transform of the temporal momentum-momentum correlation functions for dynamics with (1) and without (2) e-e interaction. Figure parts are for two densities $\left((a),(b): R_{s}=5.5 ;(c),(d): R_{s}=1.2\right)$ and temperatures $(a),(c): k_{B} T /\left|V_{0}^{e s}\right|=0.28 ;(b),(d)$ : $\left.k_{B} T /\left|V_{0}^{e s}\right|=0.45\right)$.

configurations. The results presented below are related to two different cases: (1) with e-e interaction included in the dynamics ('interacting dynamics') and (2) without e-e interaction ('non-interacting dynamics'). In both cases, the initial state fully includes all interactions.

Figure 1 presents for our model the real part of the diagonal elements of the electrical conductivity tensor versus frequency (real part of the Fourier transform of the temporal momentum-momentum correlation functions) which characterizes the Ohmic absorption of electromagnetic energy and has the physical meaning of electron conductivity. To compare the influence of electron interactions, the conductivities are given in figure 1 in the same arbitrary units. The first observation is that, in all cases, the conductivity for the non-interacting dynamics (2) has a maximum at some finite frequency related to the coherent oscillations in the time domain and vanishes at low frequency ${ }^{6}$. The latter clearly indicates Anderson

6 In fact, we observe negative values, although the real part of the conductivity has to be positive. The reason is weakly damped oscillations with a period exceeding the scale $t^{\prime}$ used in the calculation of the dynamics. To overcome this deficiency of our model one has to increase the time $t^{\prime}$ and/or to take into account the slow motion of the heavy particles, which will destroy the coherent oscillations of the light electrons trapped by the heavy particles. Additional calculations with increased $t^{\prime}$ lead to decreasing negative contributions for low frequencies, as expected. 
localization. The effect of the e-e interaction is, as shown by curves 1 , a reduction of the maximum (damping of the coherent oscillations) and, in most cases, an increase of the zero-frequency conductivity. Thus, our calculations confirm the delocalizing effect of the interactions (figures $1(a),(b),(d))$ at the considered densities. Interestingly, figure $1(c)$ is an exception: even with interactions included, the localization behaviour persists. The large oscillations in figure $1(c)$ are not the result of numerical noise; they exist in spite of very long simulation duration.

The reason for the observed behaviour is an interplay of varying strength of the e-einteraction (which is weakened with reducing $R_{s}$, i.e. from top to bottom figures) and of the magnitude of quantum effects (which grow with temperature reduction, i.e. from right to left figures). Thus, the delocalization tendency observed from figures $1(c)$ to $(d)$ is due to thermal activation which, similar to the interaction, destroys the coherence phenomena.

Our simulations qualitatively confirm analytical predictions for the low-frequency and zero temperature limit of the 1D conductivity [18]. Yet our computer power allows us to generate dynamic trajectories up to times $t^{\prime}$ equal $100 \ldots 200$ in atomic units. Thus, for small frequencies of the order $10^{-2}$, large fluctuations of the conductivity appear (see footnote 6 ), and the accuracy is not yet sufficient to extract an asymptotic frequency behaviour. On the other hand, the advantage of our computational method is that it allows us to study systematically the influence of finite temperature and of electron correlation effects on localization phenomena in a wide range of densities. We note that we have also performed simulations at lower densities and found that the delocalizing effect of the e-e interaction has also been observed at lower density up to $R_{s}=12$. At even lower densities, we expect that future simulations will yield a pinned electron Wigner crystal at weak disorder ${ }^{7}$ and Coulomb glass behaviour at strong disorder.

In summary, we have presented numerical results on the influence of Coulomb interaction on Anderson localization in a one-dimensional system. At low density $\left(R_{S}=5.5\right)$ the interaction is comparatively strong and localization is destroyed. With increasing density $R_{s}=1.2$, localization is found to persist even in the presence of Coulomb interaction. For a full understanding of the physical processes additional investigations are needed which are presently under way.

\section{Acknowledgments}

The authors thank B L Altshuler for stimulating discussions and valuable notes. VSF acknowledges the hospitality of the Graduate College 'Optoelectronics of Mesoscopic Semiconductors' and the Department of Physics of the Philipps-Universität Marburg. This work is partly supported by the Max-Planck Research Prize of the Humboldt and Max-Planck Societies, by the Deutsche Forschungsgemeinschaft under grant Bo1366-3 and by grants for CPU time at the NIC Jülich and the Rostock Linux-Cluster Fermion. IV acknowledges financial support from the Hungarian Research Fund (OTKA) under T029813, T032116 and T034832.

\section{References}

[1] Lee P A and Ramakrishnan T V 1985 Rev. Mod. Phys. 57287

[2] Belitz D and Kirkpatrik T R 1994 Rev. Mod. Phys. 64261

[3] Abrahams E, Anderson P W, Licciardello D C and Ramakrishnan T V 1979 Phys. Rev. Lett. 42637

[4] Schmid A 1991 Phys. Rev. Lett. 6080

7 In the absence of disorder, Winger crystallization is clearly found in path-integral MC simulations, see [19] and references therein. 
von Oppen F and Riedel E K 1991 Phys. Rev. Lett. 6084

Altshuler B L, Gefen Y and Imry Y 1991 Phys. Rev. Lett. 6088

[5] Pollak M 1980 Phil. Mag. 42799

[6] Zubarev D N 1974 Nonequilibrium Statistical Thermodynamics (New York/London: Plenum)

[7] Tatarskii V 1983 Sov. Phys.-Usp. 26311

[8] Filinov V S 1996 J. Mol. Phys. 88 1517-29

[9] Filinov V, Thomas P, Varga I, Meier T, Bonitz M, Fortov V and Koch S 2002 Phys. Rev. B 65165124

[10] Feynman R P and Hibbs A R 1965 Quantum Mechanics and Path Integrals (New York: McGraw-Hill)

[11] Zamalin V M, Norman G E and Filinov V S 1977 The Monte Carlo Method in Statistical Thermodynamics (Moscow: Nauka) (in Russian)

[12] Zelener B V, Norman G E and Filinov V S 1981 Perturbation Theory and Pseudopotential in Statistical Thermodynamics (Moscow: Nauka) (in Russian)

[13] Filinov V S, Bonitz M, Ebeling W and Fortov V E 2001 Plasma Phys. Control. Fusion 43743

Filinov V S, Bonitz M and Fortov V E 2000 Sov. Phys. JETP Lett. 72245

[14] Ciccotti G, Pierleoni C, Capuani F and Filinov V 1999 Comput. Phys. Commun. 121-122 452-9

[15] Filinov V, Medvedev Yu and Kamskyi V 1995 J. Mol. Phys. 85717

[16] Lozovik Yu and Filinov A 1999 Sov. Phys.-JETP 881026

[17] Sobol I M and Messer R (transl.) 1975 Monte Carlo Methods (Chicago: Univ. Chicago Publisher)

[18] Berezinskii V L 1973 Zh. Eksp. Teor. Fiz. 651251 (Engl. transl. 1974 Sov. Phys._JETP 38 620)

[19] Filinov A, Bonitz M and Lozovik Yu 2001 Phys. Rev. Lett. 863851

Filinov A, Bonitz M and Lozovik Yu 2000 Phys. Stat. Sol. (b) 221231 